\title{
Coupled multi-domain BEM and FEM for fluid-structure interaction analysis
}

\author{
V. Gnitko, V. Naumenko, U. Ogorodnyk \& E. Strelnikova \\ Institute of Mechanical Engineering Problems of the Ukrainian Academy \\ of Sciences, Ukraine
}

\begin{abstract}
The method for dynamic analysis of shell structures interacting with a liquid is presented in this paper. The coupled problem is solved using a combination of reduced boundary and finite element methods. The axisymmetric structures, such as compound shells of revolution with internal baffles and Francis turbine wheel, are under consideration. The compound shell was the model of capacity for liquid storage. The tank structure is modelled by the finite element method and the fluid region is described by the direct boundary element method. The method relies on determining the fluid pressure from the system of singular integral equations using multi-domain approach. After applying the direct boundary element formulation in every sub-domain, the final system of equations with sparse matrix was obtained by use of matching conditions in the interfaces between sub-domains. The shell is considered as thin one, and Kirghoff-Lave linear theory hypotheses are applied. The liquid is ideal and incompressible. Differential equations of transient problem are solved numerically by the RungeKutta method of 4th and 5th order. Numerical investigations of free and forced vibrations of the compound shells filled with the incompressible fluid under different loadings have been carried out. The example of the shell structure in a form of the Francis turbine sector is also under consideration. Here, a multidomain approach was compared with the hypersingular integral equation method.

Keywords: fluid-structure interaction, free and forced vibrations, boundary and finite element methods, multi-domain approach.
\end{abstract}




\section{Introduction}

Thin-walled shells are widely used in many industries including aerospace, civil, marine, petrochemical and nuclear engineering, power machine building, wind power engineering and transport. In many circumstances these shells are subjected not only to static loads but also to dynamic disturbances and filled with internal fluid. Usually they are filled with oil, flammable or toxic liquids. Such facilities are fuel tanks, liquid storage tanks, oil and propellant storage containers. The influences of both media on each other must not be neglected in stress-strength analysis of these structural elements. So the interaction between the sloshing liquid and the shell structure has been the challenging field of research in many engineering applications. In most cases, discrete techniques, such as the Finite Element Method (FEM) and the Boundary Element Method (BEM) have been employed and continuously further developed with respect to accuracy and efficiency. In fact, it did not take long until some researchers started to combine the FEM and the BEM in order to profit from their respective advantages by trying to evade their disadvantages. A detailed review on different numerical models for fluid-structure interaction can be found, e.g., in [1]. Several studies have been carried out in the different fields of sloshing liquids. Evaluation of the natural frequencies and corresponding mode shapes of liquid sloshing in a tank, linear and non-linear characters of the liquid flow, sloshing analysis in low and zero gravity, optimization and control of sloshing characteristics are some of researcher's favorite fields. Such research is needed to better understand the processes and help reduce the probability and aftermath of these tanks destruction due seismic actions or shockwaves that can lead to environmental catastrophe. The dynamic analysis of shell structures is often performed by use the finite element programs [2]. But such 3-D finite element analysis, including the contained fluid is complex and extremely time consuming. In [3-6] authors offer the approach based on using the boundary element method to the problem of natural vibrations of the fluid-filled elastic shells of revolution, as well as to the problem of natural liquid vibrations in the rigid vessels. The research findings are summarized in [7]. Multi-domain methods, or domain decomposition methods based exclusively on boundary elements have also appeared for both interior and exterior problems of fluidstructure interaction, e.g. [8-11]. The primary motivation for the multi-domain approaches for homogeneous domains stems from numerical considerations: by dividing up the original domain into smaller ones (termed macro-elements in e.g. [9]), one obtains a block-sparse algebraic system.

In this paper, the coupled problem of free and forced vibrations of shell structures interacting with the fluid is under consideration. For its solution we use combination of reduced finite and boundary element methods. The analysis consists of several stages, each represents a separate task. The frequencies and modes of shell vibrations in a vacuum are defined by the first stage. Displacement vector, that is the solution of the hydrodynamic problem, is sought as a linear combination of the natural modes of shell vibrations in vacuum. So we define the frequencies and free vibrations modes of elastic shell in the liquid 
without including the force of gravity. Then we obtain the frequencies and free vibrations modes of liquid in rigid shell under force of gravity. The latter two problems are solved using reduced BEM. The method relies on determining the fluid pressure from the system of singular integral equations using multi-domain approach. After applying the direct boundary element formulation in every subdomain, the final system of equations with sparse matrix was obtained. Then we come to second order system of differential equations for forced vibrations of the shell partially filled with a liquid and solve it numerically using Runge-Kutta method.

\section{Problem statement}

Let us consider the coupled problem for shell structure interacting with a liquid. In this study the contained liquid is assumed to be inviscid and incompressible. Governing equations of motion for the liquid-structure system subjected to disturbing force are given by

$$
\left\{\begin{array}{c}
L_{11} u+L_{12} v+L_{13} w=\rho h \frac{\partial^{2} u}{\partial t^{2}}+Q_{1}, \\
L_{21} u+L_{22} v+L_{23} w=\rho h \frac{\partial^{2} v}{\partial t^{2}}+Q_{2}, \\
L_{31} u+L_{32} v+L_{33} w=\rho h \frac{\partial^{2} w}{\partial t^{2}}+P_{l}+Q_{3} .
\end{array}\right.
$$

Here $u, v, w$ are the displacement components; $L_{i j}^{(k)}(i, j=1,2,3)$ are differential operators; $Q=\left(Q_{1}, Q_{2}, Q_{3}\right)$ is the vector of disturbing force; $\rho$ is the material density; $h$ is the shell thickness, $P_{l}$ is hydrodynamic pressure. Suppose that disturbing forces include wind, gravity and also seismic and impact waves.

Let $v_{x}, v_{y}, v_{z}$ are the fluid velocity vector components and the disturbing force $Q$ is the sum of vertical gravitational and seismic horizontal forces. Then the fluid velocity vector can be expressed in the form

$$
-\rho_{l} \frac{\partial v_{x}}{\partial t}=\frac{\partial p}{\partial x}+\rho_{l} a_{s}(t) ; \quad-\rho_{l} \frac{\partial v_{y}}{\partial t}=\frac{\partial p}{\partial y} ; \quad-\rho_{l} \frac{\partial v_{z}}{\partial t}=\frac{\partial p}{\partial z}+\rho_{l} g .
$$

Here $\rho_{l}$ is the liquid density, $a_{S}(t)$ and $g$ are the horizontal seismic acceleration and acceleration of gravity. So the liquid pressure could be expressed in the form

$$
P_{l}=p+\rho_{l} g z+\rho_{l} a_{s}(t) x .
$$

Suppose that the flow is potential. Due to the incompressibility condition the velocity potential $\phi$ satisfies the Laplace equation. The hydrodynamic pressure, according to the Cauchy-Lagrange integral, can be represented as follows

$$
\frac{p}{\rho_{l}}=-\frac{\partial \varphi}{\partial t}-g z+\frac{p_{0}}{\rho_{l}}-a_{S}(t) x
$$

where $\mathrm{z}$ is vertical coordinate of a point in the liquid. We denote a moistened surface of a shell through $S_{w}$ and a free surface as $S_{0}$. Let refer the Cartesian 
coordinate system $0 x y z$ connected with the shell. The free surface of the liquid $S_{0}$ coincides with the plane $x 0 y$ in unperturbed state.

According to [10] we obtain the following boundary value problem for defining the unknown functions $\mathbf{U}$ and $\phi$.

$$
\begin{gathered}
\boldsymbol{L U}+\boldsymbol{M} \ddot{\boldsymbol{U}}+\rho_{l} \dot{\phi}+g z+\rho_{l} a_{s}(t) x=Q ; \\
\frac{\partial \phi}{\partial n}=\frac{\partial w}{\partial t}, P \in S_{1} ; \quad \frac{\partial \phi}{\partial n}=\dot{\zeta}, P \in S_{0} ; \quad \dot{\phi}+g \zeta+\rho_{l} a_{s}(t) x=0, P \in S_{0}
\end{gathered}
$$

where $\boldsymbol{L}, \boldsymbol{M}$ are matrix operators corresponding to $L_{i j}$ and inertial components, the function $\zeta$ describes the free surface. We will seek the natural modes of shell vibrations in the fluid as following

$$
\boldsymbol{U}(x, y, z, t)=\sum_{k=1}^{m} c_{k}(t) \boldsymbol{u}_{k}(x, y, z),
$$

where functions $\boldsymbol{u}_{k}(x, y, z)$ are modes of natural vibrations in vacuum, $c_{k}(t)$ are unknown factors.

We will seek $\phi$ as a sum of two potentials $\phi=\phi_{1}+\phi_{2}$. To determine $\phi_{1}$ we obtain the following boundary value problem:

$$
\nabla^{2} \phi_{1}=0, \quad \frac{\partial \phi_{1}}{\partial n}=\frac{\partial w}{\partial t}, P \in S_{1}, \quad \frac{\partial \phi_{1}}{\partial t}=0, P \in S_{0} .
$$

Here $w(x, y, z, t)=\sum_{k=1}^{m} w_{k}(x, y, z) c_{k}(t), \quad$ and functions $\quad w_{k}(x, y, z)$ are normal modes of the shell natural vibrations in vacuum. From eqn (3) and second one from (5) it follows

$$
\phi_{1}(x, y, z, t)=\sum_{k=1}^{m} \phi_{1 k}(x, y, z) \dot{c}_{k}(t) .
$$

To determine $\phi_{1 \mathrm{k}}$ we have the following boundary value problems

$$
\nabla^{2} \phi_{1 k}=0, \quad \frac{\partial \phi_{1 k}}{\partial n}=w_{k}, P \in S_{1}, \quad \phi_{1 k}=0, P \in S_{0} .
$$

To determine $\phi_{2}$ we have to solve the problem of fluid vibrations in the rigid vessel including gravitational force

$$
\nabla^{2} \phi_{2}=0, \frac{\partial \phi_{2}}{\partial n}=0, P \in S_{1}, \frac{\partial \phi_{2}}{\partial n}=\dot{\zeta}, P \in S_{0}, \dot{\phi}_{2}+g \zeta=0, P \in S_{0} .
$$

The last equation in (8) follows from eqn (3) and represents dynamic condition on the free surface. Differentiating this equation with respect to $t$ we come to the following equation for velocity potential in the rigid vessel

$$
\ddot{\phi}_{2}+g \frac{\partial \phi_{2}}{\partial n}=0, P \in S_{0}
$$

Let us seek for the solution of this problem in the next form

$$
\phi_{2}(x, y, z, t)=e^{i \kappa t} \psi(x, y, z) .
$$


For function $\psi$ we will have the following problem of free harmonic fluid vibrations

$$
\nabla^{2} \psi=0, \frac{\partial \psi}{\partial n}=0, P \in S_{1}, \quad \frac{\partial \psi}{\partial n}=\frac{\kappa^{2}}{g} \psi, P \in S_{0} .
$$

Solving this problem one can obtain the number of eigenvalues $\kappa_{\mathrm{k}}$ and corresponding eigenfunctions $\psi_{\mathrm{k}}$. After eqn (10) is solved we are looking for function $\phi_{2}$ in the form

$$
\phi_{2}(x, y, z, t)=\sum_{k=1}^{n} \dot{d}_{k}(t) \phi_{2 k}(x, y, z) ; \phi_{2 k}(x, y, z)=\psi_{k}(x, y, z) .
$$

So we have $\phi=\phi_{1}+\phi_{2}$, where

$$
\phi_{1}(x, y, z, t)=\sum_{k=1}^{m} \phi_{1 k}(x, y, z) \dot{c}_{k}(t), \quad \phi_{2}(x, y, z, t)=\sum_{k=1}^{n} \dot{d}_{k}(t) \phi_{2 k}(x, y, z) .
$$

Then

$$
\nabla^{2} \phi=\nabla^{2} \phi_{1}+\nabla^{2} \phi_{2}=0, \quad \frac{\partial \phi}{\partial n}=\frac{\partial \phi_{1}}{\partial n}+\frac{\partial \phi_{2}}{\partial n}=\frac{\partial w}{\partial t}, P \in S_{1} .
$$

On free surface it is required

$$
\frac{\partial \phi}{\partial n}=\dot{\zeta}, P \in S_{0} ; \quad \dot{\phi}+g \zeta+a_{s}(t) x=0, P \in S_{0} .
$$

From eqns (8) and (11) we obtain the expression for the function $\zeta$ that describes the free surface perturbations

$$
\varsigma=\sum_{k=1}^{n} d_{k}(t) \frac{\partial \varphi_{2 k}(x, y, z)}{\partial n}+\sum_{k=1}^{m} \dot{c}_{k}(t) \frac{\partial \varphi_{1 k}(x, y, z)}{\partial n} .
$$

We have $\dot{\phi}_{1}=0$ from relation (7). So the equality $\dot{\phi}+g \zeta+a_{s}(t) x=0$ leads to the system of differential equations

$$
\begin{gathered}
\sum_{k=1}^{n} \ddot{d}_{k}(t) \phi_{2 k}(x, y, z)+g \sum_{k=1}^{m} \dot{c}_{k}(t) \frac{\partial \phi_{1 k}(x, y, z)}{\partial n}+g \sum_{k=1}^{n} d_{k}(t) \frac{\partial \phi_{2 k}(x, y, z)}{\partial n}+ \\
+\rho_{l} a_{s}(t) x=0 .
\end{gathered}
$$

Using for functions $\phi_{2 k}$ the relations $\frac{\partial \phi_{2 k}}{\partial n}=\frac{\kappa_{k}^{2}}{g} \phi_{2 k}, P \in S_{0}$, we obtain

$$
\sum_{k=1}^{n}\left[\ddot{d}_{k}(t)+\kappa_{k}^{2} d_{k}(t)\right] \phi_{2 k}(x, y, z)+g \sum_{k=1}^{m} \dot{c}_{k}(t) \frac{\partial \phi_{1 k}(x, y, z)}{\partial n}+\rho_{l} a_{s}(t) x=0 .
$$

Due to orthogonality of natural modes of fluid vibrations in a rigid vessel we have after dot product of the above equation and functions $\phi_{21}$

$$
\ddot{d}_{l}(t)+\kappa_{l}^{2} d_{l}(t)+\frac{g}{\left(\phi_{2 l}, \phi_{2 l}\right)} \sum_{k=1}^{m} \dot{c}_{k}(t)\left(\frac{\partial \phi_{1 k}}{\partial n}, \phi_{2 l}\right)+a_{s}(t)\left(x, \psi_{l}\right)=0, \quad l=1,2 . ., n .
$$

When functions $\phi_{1 \mathrm{k}}$ and $\phi_{2 \mathrm{k}}$ are found we substitute them in eqn (2) and obtain the following equation 


$$
L\left(\sum_{k=1}^{m} c_{k} u_{k}\right)+M\left(\sum_{k=1}^{m} \ddot{c}_{k} u_{k}\right)=-\rho_{l}\left(\sum_{k=1}^{m} \ddot{c}_{k} \phi_{1 k}+\sum_{i=1}^{n} d_{i} \phi_{2 i}+g z+a_{s}(t) x\right)+Q .
$$

Suppose that $\omega_{\mathrm{k}}, \mathrm{u}_{\mathrm{k}}$ are natural frequencies and free vibration modes of the shell in vacuum. Then the following relationships are valid

$$
\boldsymbol{L} u_{k}=\omega_{k}^{2} \boldsymbol{M} u_{k}, \quad\left(\boldsymbol{M} u_{k}, u_{j}\right)=\delta_{k j} .
$$

Considering the result of dot product of eqn (12) by $\mathrm{u}_{\mathrm{j}}$ and taking into account relationships (13), we come to the next set of $n+m$ second order differential equations

$$
\begin{gathered}
\ddot{c}_{j}(t)+\omega_{j}^{2} c_{j}(t)+\rho_{l} \sum_{k=1}^{m} \ddot{c}_{k}\left(\phi_{1 k}, u_{j}\right)+ \\
+\rho_{l}\left[\sum_{i=1}^{n} \dot{d}_{i}\left(\phi_{2 i}, u_{j}\right)+g\left(z, u_{j}\right)+a_{s}(t)\left(\rho, u_{j}\right)\right]=\left(Q, u_{j}\right), j=1, m \\
\ddot{d}_{l}(t)+\kappa_{l}^{2} d_{l}(t)+\frac{g}{\left(\phi_{2 l}, \phi_{2 l}\right)} \sum_{k=1}^{m} \dot{c}_{k}(t)\left(\frac{\partial \phi_{1 k}}{\partial n}, \phi_{2 l}\right)=0, \quad l=1,2 . ., n .
\end{gathered}
$$

So, the scheme of the solution to considered problem consists of following steps. First, we have to obtain the natural frequencies and mode shapes of free vibrations of the elastic shell in vacuum. The problem is solved using FEM. Second, it is necessary to obtain the frequencies and free vibration modes of liquid in the rigid shell under force of gravity. Then we define the frequencies and free vibration modes of elastic shell interacting with a fluid without including the force of gravity. These two problems are solved using single and multi-domain BEM. And finally we solve the set of second order differential equations using the4th and 5th order Runge-Kutta method.

\section{Multi-domain approach}

We consider the shell structure with internal baffles for damping sloshing. Sloshing is defined as the motion of free surface of a liquid in a partially filled tank or container. The inadequate slosh suppression can lead to failure of spacecrafts. For example, the early Jupiter flight was unsuccessful because the stepped-pitch program has stepping intervals near the fundamental slosh frequency and the sloshing arisen thereinafter caused the vehicle to go out of control. At first we consider the cylindrical shell with internal baffles (Figure 1). Note that multi-domain approach can be applied also to axisymmetric structures interacting with a fluid as Francis turbine (Figure 1).

The first stage is to define the velocity potential $\phi_{1}$ and therefore the partial potentials $\phi_{1 \mathrm{k}}$. To define each of these potentials we apply the direct formulation of boundary element method. To apply the multi-domain approach we divide the fluid domain into sub-domains.

According to Figure 1 we obtain the following sub-domains:

$$
\Sigma_{i}=\sigma_{i}^{1} \cup \sigma_{i}^{2} \cup \sigma_{i}^{3} ; i=1,2,3 \text {. }
$$


Here

$\sigma_{1}^{1}=S_{b} ; \sigma_{1}^{2}=S_{2} ; \sigma_{1}^{3}=S_{w 1} ; \sigma_{2}^{1}=S_{2} ; \sigma_{2}^{2}=S_{1} ; \sigma_{2}^{3}=S_{w 2} ; \sigma_{3}^{1}=S_{1} ; \sigma_{3}^{2}=S_{0} ; \sigma_{3}^{3}=S_{w 3}$.

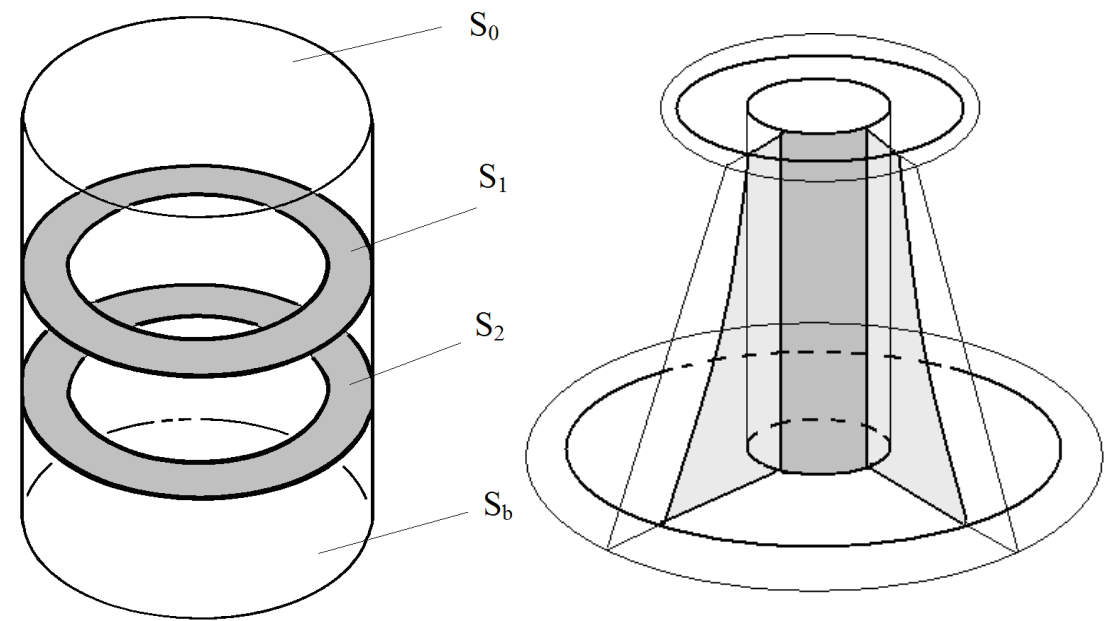

Figure 1: Cylindrical shell with internal baffles and sector of Francis turbine.

We denote as $S_{w i}(i=1,2,3)$ the parts of cylindrical shell $\mathrm{S}_{\mathrm{w}}$ between surfaces $S_{b}$ and $S_{2}, S_{2}$ and $S_{1}, S_{2}$ and $S_{0}$ accordingly. So for each partial potential $\phi_{1 \mathrm{k}}$ we obtain the following system of singular integral equations (the index $1 \mathrm{k}$ here is omitted for simplicity)

$2 \pi \varphi^{j}{ }_{l}\left(P_{0}\right)=\sum_{i=1}^{3}\left(\iint_{\sigma_{i}^{j}} \frac{\partial \varphi_{i}^{j}}{\partial n} \frac{1}{\left|P-P_{0}\right|} d \sigma_{i}^{j}-\iint_{\sigma_{i}^{j}} \varphi_{i}^{j} \frac{\partial}{\partial n} \frac{1}{\left|P-P_{0}\right|} d \sigma_{i}^{j}\right), j, l=1,2,3$.

There are 18 unknowns $\phi_{i}^{j}, \frac{\partial \phi_{i}^{j}}{\partial n},(i, j=1,2,3)$ in the system (15), but only 9 equations here. However, some of these unknowns are given at corresponding surfaces. From boundary conditions we have the following values $\frac{\partial \phi_{1}^{1}}{\partial n}, \frac{\partial \phi_{1}^{3}}{\partial n}, \frac{\partial \phi_{2}^{3}}{\partial n}, \frac{\partial \phi_{3}^{3}}{\partial n}, \phi_{3}^{2}$. On the interface surfaces $S_{1}$ and $S_{2}$ the next equalities are valid:

$$
\sigma_{1}^{2}=\sigma_{2}^{1} \Rightarrow \phi_{1}^{2}=\phi_{2}^{1} ; \quad \frac{\partial \phi_{1}^{2}}{\partial n}=\frac{\partial \phi_{2}^{1}}{\partial n} ; \quad \sigma_{2}^{2}=\sigma_{3}^{1} \Rightarrow \phi_{2}^{2}=\phi_{3}^{1} ; \quad \frac{\partial \phi_{2}^{2}}{\partial n}=\frac{\partial \phi_{3}^{1}}{\partial n} .
$$

Hence there are exactly 9 unknowns in 9 equations.

The problem of defining the partial potentials $\phi_{2 \mathrm{k}}$ by using sub-domain approach is reduced to the eigenvalue problem with a block-sparse matrix.

We use furthermore the cylindrical coordinate system and represent unknown functions as Fourier series by circumferential coordinate 


$$
w=w(r, z) \cos \alpha \theta, \quad \phi=\phi(r, z) \cos \alpha \theta .
$$

Numerical solution of the obtained systems (15) was accomplished as in $[10,13$, 14]. The boundary element method with constant approximation of unknown densities on elements was used [14]. Integration by the fluid volume was reduced to integrals along the shell meridian and along the radius of the liquid free surface. It would be noted that the only FEM analysis requires $3 \mathrm{D}$ modeling to solve this coupled problem. That leads to essentially more computer timetable and it does not allow using effectively such methods in computer monitoring problems.

\section{Analytical solution test}

We obtain the analytical solution for free vibrations of cylindrical shell fully filled with an ideal incompressible fluid. First we consider the normal modes and frequencies in vacuum. According to [15] consider the following expressions for $\mathrm{L}_{\mathrm{ij}}$ in eqn (1):

$$
\begin{gathered}
L_{11}=-\left(\delta^{2} \frac{\partial^{2}}{\partial x^{2}}+\frac{1-v}{2} \frac{\partial^{2}}{\partial \theta^{2}}\right) ; L_{12}=L_{21}=-\frac{1+v}{2} \delta \frac{\partial^{2}}{\partial x \partial \theta} ; \\
L_{13}=-L_{31}=-v \delta \frac{\partial}{\partial x} ; L_{22}=-\left(\frac{1-v}{2} \delta^{2} \frac{\partial^{2}}{\partial x^{2}}+\frac{\partial^{2}}{\partial \theta^{2}}\right) ; L_{23}=L_{32}=-\frac{\partial}{\partial \theta} \\
L_{33}=1+\tilde{h}^{2} \tilde{\nabla}^{2} \tilde{\nabla}^{2} ; \tilde{\nabla}^{2}=\frac{\partial^{2}}{\partial x^{2}}+\delta^{-2} \frac{\partial^{2}}{\partial \theta^{2}} ; \delta=\frac{R}{L} ; \tilde{h}=\frac{h R}{\sqrt{12} L^{2}} .
\end{gathered}
$$

Here $\mathrm{R}$ is the shell radius, $\mathrm{h}$ is the shell thickness and $\mathrm{L}$ is the shell length, $v$ is Poisson's ratio, $\mathrm{x}$ and $\theta$ are longitudinal and circumferential coordinates. For motion in vacuum we suppose $\mathrm{P}_{1}=0$. Consider the following boundary conditions on top and bottom of the shell:

where

$$
R_{1}+\frac{\partial M_{12}}{\partial x}=0 ; \quad \theta_{1}=0 ; u=0 ; N_{12}-\frac{1}{R} M_{12}=0,
$$

$$
\begin{gathered}
R_{1}=-D \frac{\partial}{\partial x}(\Delta w) ; M_{12}=D(1-v) \chi_{12} ; N_{12}=\frac{E h}{1-v}\left(\varepsilon_{12}-\frac{h^{2}}{12 R} \chi_{12}\right) \\
\varepsilon_{12}=\frac{1}{2}\left(\frac{\partial u}{\partial \theta}+\frac{\partial v}{\partial x}\right) ; \chi_{12}=\frac{\partial^{2} w}{\partial x \partial \theta}-\frac{1}{R} \frac{\partial v}{\partial x} ; \theta_{1}=\frac{\partial w}{\partial x}
\end{gathered}
$$

$\mathrm{E}$ is the modulus of elasticity; $\mathrm{D}$ - the bending stiffness.

Below the analytical solutions of these equations for elements of Fourier series with respect to the circumferential coordinate are represented

$$
u_{n}(t, \theta, x)=\cos n \theta \sum_{m=1}^{\infty} A_{m n}(t) \sin \frac{m \pi x}{L} ; v_{n}(t, \phi, x)=\sin n \theta \sum_{m=0}^{\infty} B_{m n}(t) \cos \frac{m \pi x}{L}
$$




$$
w_{n}(t, \theta, x)=\cos n \theta \sum_{m=0}^{\infty} C_{m n}(t) \cos \frac{m \pi x}{L} .
$$

Accordingly to described algorithm consider now the problem of evaluating the frequencies and free vibration modes of elastic shell fully filled with a liquid without including the force of gravity. It is required to define the velocity potential $\phi$ as the solution of following boundary value problem for Laplace equation in cylindrical coordinates

$$
\frac{\partial^{2} \phi}{\partial r^{2}}+\frac{1}{r} \frac{\partial \phi}{\partial r}+\frac{1}{r^{2}} \frac{\partial^{2} \phi}{\partial \theta^{2}}+\frac{\partial^{2} \phi}{\partial x^{2}}=0
$$

under boundary conditions in the form

$$
\left.\frac{\partial \phi(t, \theta, x, r)}{\partial x}\right|_{x=0, x=L}=\left.\frac{\partial u}{\partial t}\right|_{x=0, x=L}=0 ;\left.\quad \frac{\partial \phi(t, \theta, x, r)}{\partial r}\right|_{r=R}=\frac{\partial w(t, \theta, x)}{\partial t}
$$

Below we present the analytical solution of the boundary value problem (19)(20) that was obtained using the separation of variables method

$$
\phi(t, \theta, x, r)=\sum_{n=1}^{\infty}\left[\frac{\dot{C}_{0 n} r^{n}}{n R^{n-1}}+\frac{1}{\pi} \sum_{m=1}^{\infty} \frac{\dot{C}_{m n}}{m} \frac{I_{n}\left(m \pi r L^{-1}\right)}{I_{n}^{\prime}\left(m \pi r L^{-1}\right)} \cos \left(\frac{m \pi x}{L}\right)\right] \cos n \theta .
$$

It follows from eqn (21) and Cauchy-Lagrange integral (3) that the pressure at cylindrical wall is equal to

$$
P_{l}=-\rho_{l} \frac{\partial \varphi}{\partial t}=-\rho_{l} \sum_{n=1}^{\infty}\left[\frac{\ddot{C}_{0 n} r^{n}}{n R^{n-1}}+\frac{1}{\pi} \sum_{m=1}^{\infty} \frac{\ddot{C}_{m n}}{m} \frac{I_{n}\left(m \pi r L^{-1}\right)}{I_{n}^{\prime}\left(m \pi r L^{-1}\right)} \cos \left(\frac{m \pi x}{L}\right)\right] \cos n \theta .
$$

Here $I_{n}(z)$ are modified Bessel functions of the first kind. Hereinafter we use expression (21) for comparison between analytical and numerical solutions.

\section{Numerical results}

First we consider the cylindrical shell fully filled with a fluid and apply the boundary integral equation technique for determining the fluid pressure. The single-domain and two-domain BEM were used. The cylindrical shell without baffles has following parameters: the shell radius $R=1.0 \mathrm{~m}$, the length $\mathrm{L}=2.0 \mathrm{~m}$. To validate numerical algorithm we have solved the singular integral eqns (15) using two methods: by single-domain and two-domain BEM approach. Here the normal modes in the form $w_{k}(x, \theta)=\cos \left(k \pi x L^{-1}\right) \cos \theta ; k=1,2$ were used. The following analytical expressions for the potentials $\phi_{\mathrm{k}}$ matched to these modes accordingly to eqn (21)

$$
\phi_{k}(\theta, x)=\cos \theta \cos \left(k \pi x L^{-1}\right) I_{1}(k \pi \delta)\left[k \pi I_{1}^{\prime}(k \pi \delta)\right]^{-1}, \quad k=1,2 .
$$

Here $\delta$ was defined in eqns (17). According to the concept of [10] the integration by the fluid volume was reduced to integrals along the shell meridian and the 
liquid free surface radius, so for each harmonic mode $\operatorname{cosn} \theta$ we obtain the separate system of singular integral equations.

The comparison between numerical and analytical results is shown in Figure 2.

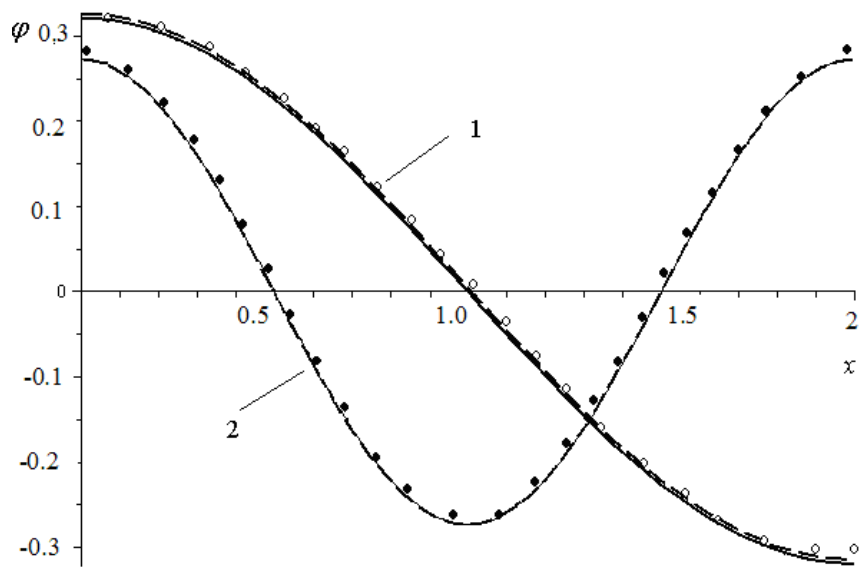

Figure 2: Numerical and analytical solutions.

Here the solid lines denote the analytical solutions in the form $\varphi_{k}(x)=\cos \left(k \pi x L^{-1}\right) I_{1}(k \pi \delta)\left[k \pi I_{1}^{\prime}(k \pi \delta)\right]^{-1} ; k=1,2, \quad$ numbers $\quad 1$ and 2 correspond to index $\mathrm{k}$; by dot lines the single-domain BEM solutions are shown that were obtained at $\mathrm{n}_{1}=\mathrm{m}_{1}=10$ boundary elements along the shell meridian and the free surface radius, and dash lines are solutions obtained by using twodomain BEM with $\mathrm{n}_{1}=\mathrm{m}_{1}=10$ boundary elements in each domain. Note that using two-domain BEM does not increase the dimension of matrices in numerical simulation.

The next numerical result is concerned with defining the added mass matrix for a mechanical structure interacting with an incompressible fluid. The Francis turbine wheel was considered here as an example of such fluid-structure interaction. Two methods were implemented in numerical simulation. First of them relies on hypersingular integral equations obtained from the indirect boundary element formulation. The infinite liquid volume was considered here and the blades were modeled by thin surfaces. The implementation of boundary integral equation technique led to following system of hypersingular equations

$$
\frac{1}{4 \pi} \sum_{i=1}^{N_{b}} \iint_{\Omega_{i}} \gamma_{i}\left(\boldsymbol{x}_{i}\right) \frac{\partial^{2}}{\partial n(\boldsymbol{x}) \partial n\left(\boldsymbol{x}_{0 j}\right)} \frac{1}{\left|\boldsymbol{x}_{j}-\boldsymbol{x}_{0 j}\right|} d \Omega_{i}=\frac{\partial w\left(\boldsymbol{x}_{0 j}\right)}{\partial t} ; j=1,2, \ldots N_{b},
$$

where $\mathrm{N}_{\mathrm{b}}$ - the number of blades in Francis turbine wheel, $\gamma_{i}(\boldsymbol{x}), i=1,2, \ldots, N_{b}$ are unknown densities; each of them is proportional to the pressure drop on both sides of the blade. Another approach to numerical simulation of the pressure on blades is based on using the direct boundary element formulation and multi- 
domain method. Here the system of singular integral equations analogical to (15) was solved. The advantage of this approach consists in possibility to consider the finite volume of fluid. In Table 1 the comparison between experimental data and numerical results obtained by two methods is presented. These data were obtained for the model of the wheel of Francis turbine in Piedra del Aguila power plant (Argentina).

Table 1: $\quad$ Comparison between numerical and experimental frequencies.

\begin{tabular}{|c|c|c|c|c|}
\hline & \multicolumn{2}{|c|}{ Numerical results, Hz } & \multicolumn{2}{c|}{ Experimental data, Hz } \\
\hline $\begin{array}{c}\text { Frequency's } \\
\text { number }\end{array}$ & $\begin{array}{c}\text { Hyper- } \\
\text { singular } \\
\text { integral } \\
\text { equation }\end{array}$ & $\begin{array}{c}\text { Direct } \\
\text { formulation, } \\
\text { multi-domain } \\
\text { BEM }\end{array}$ & $\begin{array}{c}\text { I.I. Polzunov SDA } \\
\text { on Research and } \\
\text { Design of Power } \\
\text { Equipment }\end{array}$ & $\begin{array}{c}\text { OJSC } \\
\text { "Turboatoom" }\end{array}$ \\
\hline 1 & 24.00 & 22.2 & 22.5 & 21.6 \\
\hline 2,3 & 29.20 & 28.7 & 28.5 & 28.5 \\
\hline 4,5 & 31.50 & 32.3 & 31.1 & 32.7 \\
\hline 6,7 & 37.00 & 35.3 & 33.3 & 37.2 \\
\hline 8 & 52.50 & 45.3 & & 40.2 \\
\hline
\end{tabular}

Within the framework of this research the numerical simulation was also accomplished for rigid end elastic cylindrical, conical and hemi-spherical shells subjected to impulse and seismic loads. These problems were investigated in [10, 14] by using single-domain BEM. The new results were obtained with multidomain BEM. Comparison between results testifies the efficiency and accuracy of multi-domain approach.

\section{Conclusions}

The numerical procedure based on a coupling the finite element formulation and the boundary element method is developed for numerical analysis of fluidstructure interaction. We introduce the representation of the velocity potential as the sum of two potential corresponding to problem of fluid free vibrations in the rigid shell under force of gravity and to problem of elastic shell free vibrations with fluid without including the gravitational component. Integration by the fluid volume is reduced to integrals along the shell meridian and along the radius of the liquid free surface. It is the basic advantage of our method based on a combination of the boundary integral equation method with single and multidomain approach, finite element method, expansion into Fourier series and mode superposition. The shell structure in a form of the Francis turbine sector was also under consideration. Here multi-domain approach was compared with hypersingular integral equation method.

\section{Acknowledgement}

The authors gratefully acknowledge our collaborator on STCU Projects Professor Carlos Brebbia for his constant support and interest in our research. 


\section{References}

[1] Amabili, M. and Païdoussis, M. P., Review of studies on geometrically nonlinear vibrations and dynamics of circular cylindrical shells and panels, with and without fluid-structure interaction. Applied Mechanics Review, 56(4), pp. 349-381, 2003.

[2] Sanchez-Sanchez, H., Cortes, S.C., Seismic response of cylindrical tanks for oil// Proc. of $14^{\text {th }}$ World Conference on Earthquake Engineering, Beijing, China, 2008.

[3] Yu, G. Y., Lie, S. T. and Fan, S. C., Stable boundary element method/finite element method procedure for dynamic fluid-structure interaction, A.S.C.E. Journal of Engineering Mechanics, 128, pp. 909-915, 2002.

[4] Kumar, V. and Ganesan, N., Dynamic analysis of conical shells conveying fluid. Journal of Sound and Vibration, 310(1-2), pp. 38-57, 2008.

[5] Zhang, Y.L., Gorman, D.G. and Reese, J.M., Vibration of prestressed thin cylindrical shells conveying fluid. Thin-Walled Structures, 41, pp. 1103$1127,2003$.

[6] Chen, Y.H., Hwang, W.S. and Ko, C.H., Numerical simulation of the threedimensional sloshing problem by boundary element method. Journal of the Chinese Institute of Engineers, 23(3), pp. 321-330, 2000.

[7] Recent Advances in Boundary Element Methods A Volume to Honor Professor Dimitri Beskos Manolis, George; Polyzos, Demosthenes Eds., XXXVIII, p. 470, 2009.

[8] Bielak J., MacCamy R.C., Symmetric finite element and boundary integral coupling methods for fluid-solid interaction, Q. Appl. Math. 49, pp. 107$119,1991$.

[9] Zeng X., Kallivokas L.F., Bielak J., Stable localized symmetric integral equation method for acoustic scattering problems J. Acoust. Soc. Am. 91(5), pp. 2510-2518, 1992.

[10] Gnitko V., Marchenko U., Naumenko V., Strelnikova E., Forced vibrations of tanks partially filled with the liquid under seismic load. Proc. of XXXIII Conference "Boundary elements and other mesh reduction methods" WITPress, Transaction on Modeling and Simulation, pp. 285-296, 2011.

[11] Ravnik, J., Škerget, L., Žunič, Z., Combined single domain and subdomain BEM for 3D laminar viscous flow. Engineering analysis with boundary elements, 33(3), pp. 420-424, 2009.

[12] Ravnik, J., Škerget, L., Žunič, Z. Velocity-vorticity formulation for 3D natural convection in an inclined enclosure by BEM. Int. J. Heat Mass Transfer, 51(17/18), pp. 4517-4527, 2008.

[13] Brebbia, C.A., Telles, J.C.F. and Wrobel, L.C. Boundary Element Techniques, Springer-Verlag: Berlin and New York, 1984.

[14] Ventsel, E. S. Naumenko, V., Strelnikova, E., Yeseleva, E., Free vibrations of shells of revolution filled with a fluid Engineering analysis with boundary elements, 34(10), pp. 856-862, 2010.

[15] Ventsel E., Krauthammer, T., Thin plates and shells: Theory, Analysis and Applications. Marcel Dekker, Inc. 2001. 